\title{
Ionospheric Tomography Using GNSS Reflections
}

\author{
Josep Marco Pallarés, Giulio Ruffini, and Leonardo Ruffini
}

\begin{abstract}
In this paper, we report a preliminary analysis of the impact of Global Navigation Satellite System Reflections (GNSS-R) data on ionospheric monitoring over the oceans. The focus will be on a single polar Low Earth Orbiter (LEO) mission exploiting GNSS-R as well as Navigation (GNSS-N) and Occultation (GNSS-O) total electron content (TEC) measurements. In order to assess impact of the data, we have simulated GNSS-R/O/N TEC data as would be measured from the LEO and from International Geodesic Service (IGS) ground stations, with an electron density (ED) field generated using a climatic ionospheric model. We have also developed a new tomographic approach inspired by the physics of the hydrogen atom and used it to effectively retrieve the ED field from the simulated TEC data near the orbital plane. The tomographic inversion results demonstrate the significant impact of GNSS-R: three-dimensional ionospheric ED fields are retrieved over the oceans quite accurately, even as, in the spirit of this initial study, the simulation and inversion approaches avoided intensive computation and sophisticated algorithmic elements (such as spatio-temporal smoothing). We conclude that GNSS-R data over the oceans can contribute significantly to a Global/GNSS Ionospheric Observation System (GIOS).
\end{abstract}

Index Terms-Global Navigation Satellite System (GNSS), Global Navigation Satellite System Reflections (GNSS-R), ionosphere, Low Earth Orbiter (LEO), tomography.

\section{INTRODUCTION}

I ONOSPHERIC electron content measurements provide an important element for space weather research and operations. Adverse conditions in the space environment can cause disruption of satellite operations, communications, navigation and electric power distribution grids, leading to a variety of socio-economic losses. Knowledge of ionospheric electron content is also very important for the operation of space radar systems [e.g., space altimetry, including Global Navigation Satellite System Reflections (GNSS-R)]. The initial focus of this paper will be on better coverage of data-void or data-sparse regions (e.g., data over the oceans, complementary data). Little data on ionospheric electron content is presently available over the oceans, although this situation will be mitigated by global positioning system (GPS) occultation measurements (e.g., from CHAMP and COSMIC, the U.S./Taiwan constellation), and the vertical character of GNSS-R soundings together with their availability over water (and perhaps ice and land) covered areas will be able to fill these gaps.

It is well known that the atmosphere affects the propagation of radio signals. The neutral troposphere and the ionosphere have an impact on ranging measurements from radar systems, and

Manuscript received March 15, 2004; revised October 26, 2004. This work was supported in part by the GIOS ESA/ESTEC Starlab Project Starlab/CO/0001/02.

The authors are with Starlab Barcelona, Camí de l'Observatori s/n, 08035 Barcelona, Spain (e-mail: josep.marco@starlab.es).

Digital Object Identifier 10.1109/TGRS.2004.841253 both have been an object of intense research exploiting the fact that GPS (L-band) signals are susceptible to the atmospheric gas composition and plasma distribution. This, tied to the high precision of the GPS system, has opened a wide door to study atmospheric phenomena. In fact, it has been an important goal for the GNSS research community to test the limits of the geophysical measurement techniques derived from this technology.

Both GPS and the forthcoming European Galileo are designed for precise navigation as multifrequency systems: the ionospheric contribution to the delay can then be measured and removed by making use of its dispersive nature.

Because of the existence of ionized free electrons, the ionosphere adds a delay of a few meters to the GNSS signal (L-band). The exact amount depends on the electron density along the ray link path and on which of the GNSS available frequencies is considered (e.g., in GPS, $f_{1}=1.57542 \mathrm{GHz}$ and $f_{2}=1.22760 \mathrm{GHz}$ ). As mentioned, the dispersive nature of this phenomenon is exploited to measure the integrated free electron content delay accurately, and, if needed, to remove it from the measurements (as in dual frequency GPS, for example). Consider a signal traveling at time $t$, between a given satellite and receiver, and let $I=\int_{\text {ray }} \rho(\bar{x}) d l$ be the integrated electron density or total electron content (TEC) along the ray traversed on by the signal (in electrons per square meter). Then the delay at frequency $f_{i}$ is modeled by

$$
\begin{aligned}
D_{i}=D_{\mathrm{sl}}(t)+I \alpha / f_{i}^{2} & +T \\
& +c_{\mathrm{sat}}^{i}+c_{\mathrm{rec}}^{i}+\text { noise }+\left(D^{i}-D_{\mathrm{sl}}\right)
\end{aligned}
$$

where $\alpha=40.30 \mathrm{~m}^{3} / \mathrm{s}^{2}, D^{i}$ is the geometric length of the real ray, $D_{\mathrm{sl}}$ is the length of the ray if it traveled in a straight line (in the vacuum), $T$ models other frequency-independent terms, and $c_{\mathrm{sat}}^{i}$ and $c_{\mathrm{rec}}^{i}$ are the instrumental biases (which are assumed to remain constant in relatively long timescales). The last term is the difference between the length of the real ray and the length of the ray if it propagated in the vacuum and is also small for nongrazing geometries.

Ionospheric delay on GPS is usually expressed in electrons per square meter divided by $10^{16}$ (TEC units or TECU). Electron density (ED) is usually expressed by the number of free electrons per cubic meter. Typical peak values of ED are of the order of $10^{12}$ electrons per cubic meter (i.e., Tera electrons $/ \mathrm{m}^{3}$ ) and are found around $300 \mathrm{~km}$ of altitude.

A standard approach for 3-D tomography of the ionosphere is based on the voxel basis representation [7]. As with other approaches, an important problem in voxel tomography is that it is in general an ill-determined problem: the data available for inversion are typically not sufficient to uniquely specify a solution to the resolution desired. This problem is compounded 
by the fact that data availability is typically nonhomogeneous. Several methods can be used to address this problem [10]. The problem in voxel tomography is linear, of the form $y=A x$, where the matrix A is the "integration matrix" and has as many rows as there are data measurements (one for each ray, or satellite-receiver link) and as many columns as there are unknowns in the problem (one for each voxel plus the bias constants). Its entries, then, are the lengths of the ray portions "spent" in each voxel, plus a 1 or 0 depending on the satellite and receiver involved (in the bias sector of A).

The equation $y=A x$ can be recast into a minimization problem: find $x$ so that $F=\|y-A x\|_{C}^{2}=(y-A x)^{T} C^{-1}(y-$ $A x)$ is minimized, and where $\mathrm{C}$ is the measurement covariance matrix (the notation $\|x\|_{M}$ means $x^{T} M^{-1} x$ ). In general, there will be many solutions to this problem, however, and this is reflected by the fact that $A^{T} C^{-1} A$ is singular. The approach typically taken to solve this problem is to add a reasonable constraint, and define a new functional to minimize, $F_{r}=\| y-$ $A x\left\|_{C}^{2}+\right\| x \|_{B}^{2}$, where $C$ and $B$ and the corresponding covariance and constraint matrices. These constraints are normally smoothing constraints. This is a natural choice: if measurements are missing from some portion of the 3-D grid we ask that the solution effectively interpolate $\mathrm{x}$ using the data from other places. Thus, the grid itself does not set the minimal resolution of the solution, only the maximal one. The effective resolution can be coarser depending on data availability. It is important to note that the constraint will have strong effect in areas of low data availability, while in areas with abundant data they will not interfere too much. The effective resolution of the system is thus not homogeneous, but will vary depending on the area's data availability.

Voxel tomography is a rather computationally intensive method, typically involving thousands of unknowns. A promising alternative is the ingestion of GPS data into models. For example, in [6], GNSS TEC data was ingested into the parameterized ionospheric model (PIM, a climatic model: http://www.cpi.com/products/pim/) using a simple least squares approach, and good fits to GPS data were obtained tuning just a few parameters (resulting in $40 \mathrm{~cm}$ of postresidual mean, compared to $30 \mathrm{~cm}$ using full-blown tomographic methods involving many more parameters). The main disadvantage of model-based tomographic approaches is that they will inevitably bias the solution toward the model, of course.

In this paper, we will use another method, based on a nonlocal basis adapted to a spherical context. The method is suitable for the simulation task at hand, where data are primarily available on a localized region.

The possibility of using GNSS-R ionospheric TEC data is of high interest: GNSS-R can potentially provide a high number of near-vertical, or at least oblique, bi-TEC measurements over the oceans (the term bi-TEC refers to the double-link nature of the TEC integral, due to the reflection process), an important missing piece in a future GNSS Ionospheric Monitoring System. Ionospheric electron content data measured along vertical directions enhances considerably the accuracy of tomographic models (voxel or model based), since it complements occultation soundings, which are of horizontal nature. Little data with these characteristics is presently available over the oceans, and the vertical character of GNSS-R soundings together with their availability over water (and perhaps ice or land) covered areas will be able to fill these gaps, complementing occultation data. Finally, we mention that GNSS-R altimetric applications will necessitate the correction of ionospheric effects, and this is likely to be done through the development of tomographic models as described below.

In previous work [5], the use of spaceborne GNSS-R data to sound the ionosphere was investigated (a possibility already discussed in [2]), with a focus on accounting for the error budget associated to ionospheric GNSS-R.

\section{Simulating GNSS TEC DATA}

The goal in this feasibility study was to perform tomography of the ionosphere using combined GNSS-R/O/N LEO and ground data to produce realistic 3-D models of the electron density content of the atmosphere. An important ingredient was the use of the most recent GNSS-R error budget, based on recent experimental and theoretical work [4].

To assess the impact of GNSS-R data, we simulated the ionosphere using PIM and measured TEC using a virtual GPS constellation (in its current configuration) and a single polar LEO satellite, as well as relying on the current IGS network for ground measurements. A polar orbiter is a good choice, as discussed in the [3], providing global coverage for many applications (such as mesoscale altimetry or "scatterometry").

Note, however, that for the purposes of ionospheric monitoring the conditions for observation are rather different than for other types of earth observation. This is because the ionosphere is quasistatic in an inertial frame. Therefore, a single LEO will only be able to sample a rather fixed slice of the ionosphere in short time scales (e.g., days). Ground stations and filtering techniques can be used to propagate the slice solution elsewhere, but at a price in precision.

As mentioned, only one LEO orbit was implemented in our simulation. This is because the goal of this initial study was limited to the demonstration of the impact of GNSS-R data in the Global/GNSS Ionospheric Observation System (GIOS), and the near-future outlook of GNSS-R involves a single satellite mission. As discussed, GNSS-R data are expected to have the biggest impact over the oceans, where there is presently no data, and where GNSS-O data will only able to provide horizontal TEC measurements. For the purposes of demonstration, we aimed to show that within a given slice over the oceans we could produce a sensible ED solution (see Fig. 1).

In addition, as the purpose of the study was to demonstrate the impact of the data at relatively low spatial resolutions, virtual TEC data were generated with a rather low rate (1-3 min). In reality, much more data will be available - the spatial sampling will be denser. Nonetheless, we believed (correctly, as it turned out), that even with this meager data rate we could demonstrate the impact of the measurements.

In the simulation, we have considered the following sources of ionospheric (slant) TEC data:

- GNSS-G data: phase-quality TEC data as produced by the present $300+$ IGS stations on the ground; 


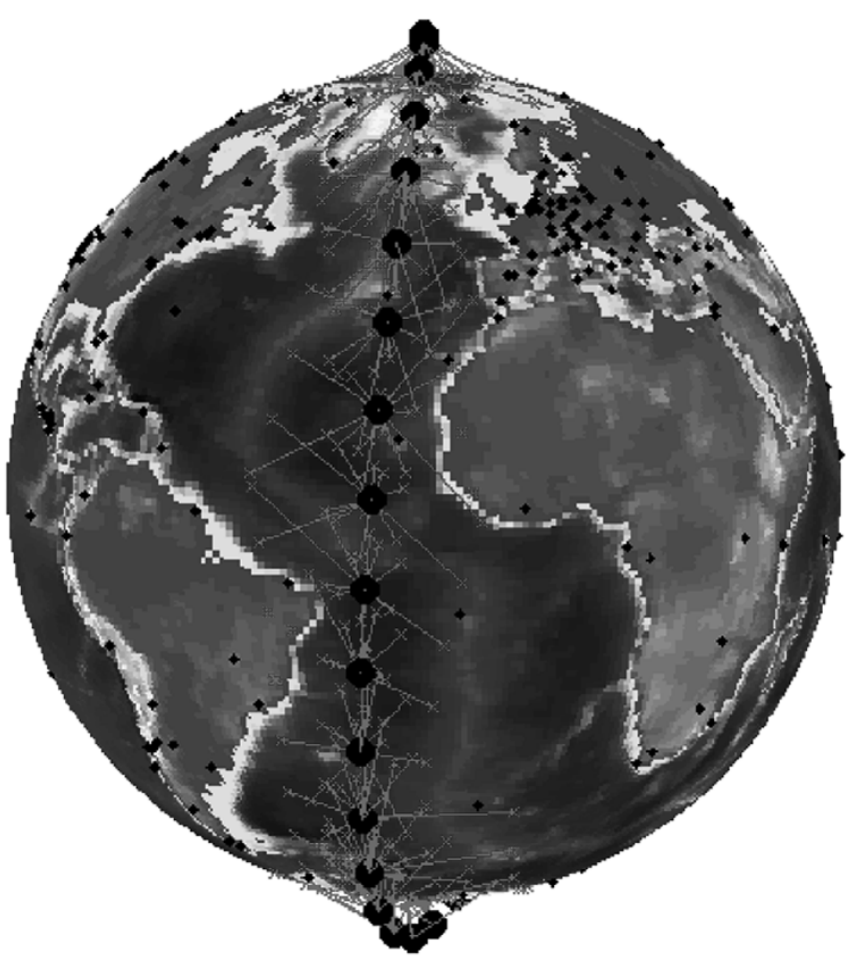

Fig. 1. LEO positions with a cadence of 3 min (the orbit positions are shown as large black dots). The specular links (over the oceans) are shown in as lines. Ground stations are shown as small black dots. Land reflections are identified and neglected (not linked).

- GNSS-O/N data: phase-quality TEC data as produced from GNSS LEO Occultation and Navigation links;

- GNSS-R data: this denotes reflected GPS-to-ground-toLEO bi-TEC data obtained from code-ranging (not using the phase).

Gaussian noise was added to the simulated GNSS-R TEC data: an error of ten TECUs standard deviation was added for a measurement equivalent to $10 \mathrm{~s}$ of averaging (a rather conservative assessment), as we discuss below. We recall here that for the GPS ionospheric combination $\left(L_{I}=D_{1}-D_{2}\right.$, measured in meters) 1 TECU is equivalent to $10.5 \mathrm{~cm}$ of $L_{I}$ delay, and that typical vertical TEC is between 0 and 50 TECU.

The other types of GNSS TEC data are in comparison not very affected by noise (they present sub-TECU noise levels), thanks to the higher SNR and the precision of phase ranging, and no noise was added.

According to present models and experiments, GNSS-R L1 code bistatic ranging data has an intrinsic noise of less than $0.5 \mathrm{~m}$ (about five TECU) after $1 \mathrm{~s}$ of integration, with the assumption of a reasonably large LEO antenna ( $\sim 27 \mathrm{~dB})$ (see [4], [6], and [8]). With such a mission scenario, the noise level in our simulations is therefore amply justified, even considering for the slightly worse L2 (unencrypted) error budget. We believe, and we assumed that L1 C/A code is a realistic representative of other GNSS signals, present or future, without further claim for a very accurate simulation. Even in the context of a low antenna gain GNSS-R mission (with a gain of about $15 \mathrm{~dB}$ ), dual frequency code measurements would be of value for ionospheric applications.
Using dual frequency code pseudoranges, the aforementioned error budget models predict that with a $15 \mathrm{~dB}$ mission the ionospheric combination double-slant delays could be measured to better than $2 \mathrm{~m}$ after $1 \mathrm{~s}$ of integration, leading to vertical TEC measurements of about 15 TECU accuracy after $1 \mathrm{~s}$ or about 3 TECU after 20-s averaging-still well within the bounds of our simulated noise.

In the simulations, we focused on the code ranging scenario. GNSS-R single frequency phase tracking from space is likely to be very difficult except in extremely rare calm sea conditions and/or grazing incidence. We note in passing, however, that phase tracking of the ionospheric combination, $L_{I}$, may be possible. Simulations based on realistic ocean surface models indicate that the phase of the ionospheric combination is very well behaved after scattering and not very sensitive to sea state conditions [5]. Although this may seem surprising at first, it is somewhat expected, as the ionospheric combination can be interpreted to synthesize an infinite synthetic wavelength (an extreme form of wide-laning). Longer wavelengths are naturally less sensitive to surface roughness, and previous work demonstrates that such synthetic wavelengths have similar properties (see [5] and references therein). This could imply that accurate GNSS-R ionospheric phase measurements will be feasible from space, a possibility that warrants future research.

With regards to the 3-D ED ionospheric model (the reference "truth"), PIM was used with a resolution of about $10^{\circ}$. Developed integration routines used the model to produce virtual TEC along the required emitter-receiver links.

\section{TOMOGRAPHIC APPROACH: THE H-REPRESENTATION}

As discussed in the Introduction, we used a new representation (which we call the H-representation) for tomographic inversion, based on the solutions to the Schrödinger equation for the hydrogen atom.

This representation is complete (with a caveat, see below) and nonlocal. The second point means that if data are available at only specific regions of the ionosphere, all the coefficients in the representation will contribute to the fit in the data-rich area. This allows for a good fit where there is data at the expense of sparsely sampled regions. This "economy" was very useful in the present case, since, as mentioned, most of the data generated was available near the LEO orbital ring. Thus, with a relatively small number of coefficients we expected a good fit of the data.

The H-Representation is well suited to a spherical coordinate system and also offers the advantage of easy integration of smoothing terms to account for data scarcity. We also note that the distribution of electrons in the ionosphere shares some resemblance to the electronic probability density in the hydrogen atom (including the existence of a density peak height).

The basis used is of the form

$$
\begin{aligned}
& \mathrm{ED}(r, \theta, \phi)=\sum_{n} \sum_{l} \sum_{m} a_{\mathrm{nlm}} \mathrm{ED}_{\mathrm{nlm}} \\
& \mathrm{ED}_{\mathrm{nlm}}(r, \theta, \phi)=R_{\mathrm{nl}}(r) Y_{\mathrm{lm}}(\theta, \phi)
\end{aligned}
$$


(with $r$ the altitude over the ground) as in the Schrödinger solution to hydrogen atom. Here, $Y_{\mathrm{lm}}$ are the spherical harmonics basis functions, and $R_{\mathrm{nl}}$ the radial basis functions

$$
R_{\mathrm{nl}}(r)=c_{\mathrm{nl}} e^{-\rho / 2} \rho^{l+1} L_{n-l-1}^{2 l+1}
$$

where $c_{\mathrm{n}, 1}$ are normalization coefficients that ensure that

$$
\int_{0}^{25000 \mathrm{~km}}\left(c_{\mathrm{n}, 1} e^{-\rho / 2} \rho^{l+1} L_{n-l-1}^{2 l+1}(\rho)\right)^{2} d r=1 .
$$

Similarly, $L_{n-l-1}^{2 l+1}$ are the Laguerre polynomials defined by the equation

$$
L_{n}^{k}(x)=\sum_{m=0}^{n}(-1)^{m} \frac{(n+k) !}{(n-m) !(k+m) ! m !} x^{m}
$$

and $\rho$ as defined as

$$
\rho=\frac{2 r}{n a_{0}}
$$

(see [1] and [9]). In the hydrogen atom, $a_{0}$ corresponds to the Bohr radius. In our case, this is a parameter to determine to optimize the fit. Our simulations show that good fits are given by $a_{0}$ between $20-30 \mathrm{~km}$. This, however, will generally depend on the largest value of $n$ allowed (as discussed below).

Note also that in (4) we have added an additional $\rho$ term as compared with the hydrogen atom wavefunction solutions to force the basis functions to be zero at zero altitude (a desirable feature).

Now, we are to solve the following system (we ignore here the covariance matrix, as we assumed a simple multiple of the identity)

$$
y=A \cdot x
$$

where each row in y (the measured slant TEC) is computed from

$$
y_{n}=\gamma \int \cdot E D \cdot d l
$$

(the line integral is computed along the data link). Inserting the definition in (2), we have

$$
\begin{aligned}
& y=\int \sum_{n} \sum_{l} \sum_{m} a_{\mathrm{nlm}} \mathrm{ED}_{\mathrm{nlm}}(r, \theta, \phi) d l \\
& y=\sum_{n} \sum_{l} \sum_{m} a_{\mathrm{nlm}} \int \mathrm{ED}_{\mathrm{nlm}}(r, \theta, \phi) d l .
\end{aligned}
$$

In the language of the linear system discussed above, the array $x$ here is the array of the coefficients $a_{\mathrm{nlm}}$ (the unknowns), and the matrix $A$ is the matrix of integrals, which is of the form shown at the bottom of the page, while the array $x$ has the form

$$
x=\left(\begin{array}{l}
a_{100} \\
a_{200} \\
a_{210} \\
a_{211} \\
a_{211}
\end{array}\right) .
$$

So, given the TEC for each ray link and the corresponding matrix $A$, we are to solve

$$
x=\left(A^{T} A\right)^{-1} \cdot A^{T} y .
$$

The inversion can be regularized using smoothing terms if a high number of coefficients (more than the available measurement equations) is desired.

We note that the number of unknowns increases following the relation

$$
N=\sum_{i=1}^{n} i^{2} .
$$

For $n=1$, there is only one coefficient, for $n=2$ there are five coefficients, for $n=3$, there are 14 coefficients, etc.

\section{TOMOGRAPHIC RESULTS}

The H-representation appeared to provide an efficient way to represent the solution space.

With as little as $n=5$ (55 coefficients), we obtained a data fit of 7 TECU under the LEO track, using only LEO data. Slant data fit residuals globally were of 13 TECU with the addition of IGS ground data (the increase is natural, as we are adding

$$
\begin{aligned}
& A=\left(\begin{array}{ccc}
\int R_{10}(r) Y_{00}(\phi, \theta) d l_{1} & \int R_{20}(r) Y_{00}(\theta, \phi) d l_{1} & \int R_{21}(r) Y_{10}(\theta, \phi) d l_{1} \\
\int R_{10}(r) Y_{00}(\theta, \phi) d l_{2} & \int R_{20}(r) Y_{00}(\theta, \phi) d l_{2} & \int R_{21}(r) Y_{10}(\theta, \phi) d l_{2} \\
\ldots & \ldots & \ldots \\
\int R_{10}(r) Y_{00}(\theta, \phi) d l_{n} & \int R_{20}(r) Y_{00}(\theta, \phi) d l_{n} & \int R_{21}(r) Y_{10}(\theta, \phi) d l_{n} \\
\ldots & \ldots & \ldots
\end{array}\right. \\
& \operatorname{Re}\left(\int R_{21}(r) Y_{11}(\theta, \phi) d l_{1}\right) \quad \operatorname{Im}\left(\int R_{22}(r) Y_{11}(\theta, \phi) d l_{1}\right) \quad \cdots \\
& \begin{array}{ccc}
\operatorname{Re}\left(\int R_{21}(r) Y_{11}(\theta, \phi) d l_{2}\right) & \operatorname{Im}\left(\int R_{22}(r) Y_{11}(\theta, \phi) d l_{2}\right) & \ldots \\
\ldots & \ldots & \ldots
\end{array} \\
& \left.\begin{array}{ccc}
\operatorname{Re}\left(\int R_{21}(r) Y_{11}(\theta, \phi) d l_{n}\right) & \operatorname{Im}\left(\int R_{22}(r) Y_{11}(\theta, \phi) d l_{n}\right) & \ldots \\
\ldots & \ldots & \ldots
\end{array}\right)
\end{aligned}
$$




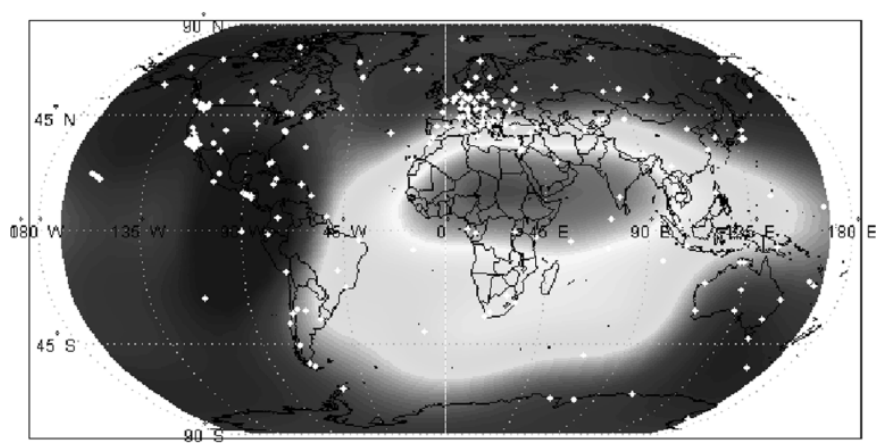

(a)

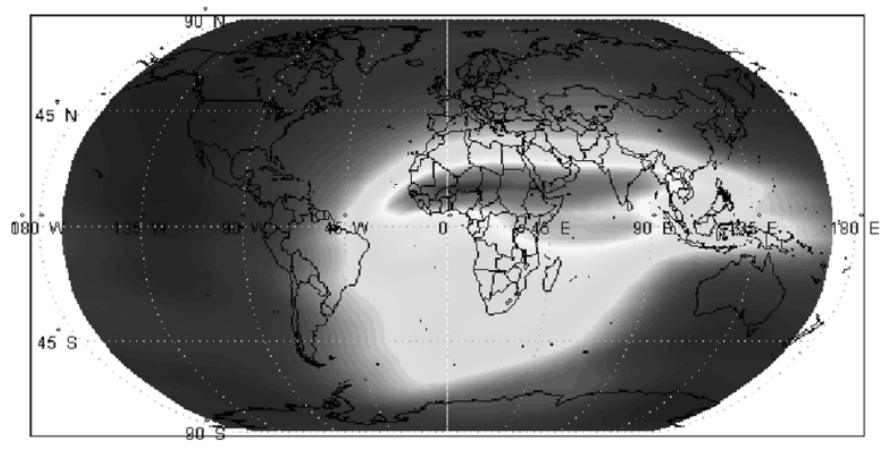

(b)

Fig. 2. (a) Recovered vertical TEC with $n=8$ (204 coefficients, $a_{o}=$ $20 \mathrm{~km}$ ), using ground data (IGS stations appear as white dots) and all LEO data (providing more than 10000 measurements). Residual variance was of 6.3 (slant) TECU, mainly due to model "quantization." (b) PIM ionospheric vertical TEC is shown (i.e., the vertical TEC corresponding to the virtual ED used for data generation).

measurements, or equations, while maintaining the number of parameters to fit). The addition of ground data from a few stations provided a better global ED solution, as expected.

Figs. 2 and 3 display the results of the $n=8$ TEC fit including the orbital ring ED solution.

The main results are summarized in quantitative form in Table I. Note that the fourth column refers to the data fit residuals (data minus model). Such residuals will always increase (or at best stay the same) with the addition of new data. On the contrary, ED model residuals (last column) refer to "truth" minus estimate of the ED solution, which should have the opposite behavior (the more data the better the solution).

As expected, as the order of $n$ and the number of coefficients increases, a better fit results. It is clear that using GNSS-R data improves the ED results on the orbital ring area (see Fig. 3 for a picture of the ring slice), as expected, although a bigger impact is expected at higher $n$. Also, the $a_{0}$ parameter must be somewhat tuned to avoid putting weight above the area of interest.

In the last four rows (bold), we show preliminary solutions with a smoothing constraint penalizing high $n$ components of the solution (this is done by adding a constraint penalizing solutions with weight in high $n$ basis functions, of the form $\left.\lambda \operatorname{diag}\left[n^{2}\right]\right)$. The corresponding functional to this constraint is

$$
F=(y-A x)^{T} C^{-1}(y-A x)+\lambda \operatorname{diag}(N)
$$

where $N$ is a matrix that contains in its diagonal the $n^{2}$ values of the corresponding base function.

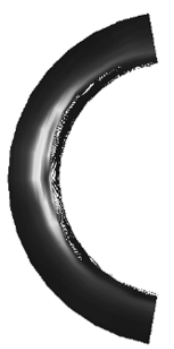

(a)
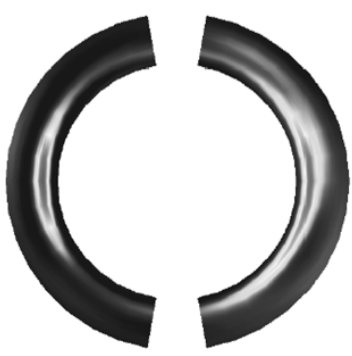

(c)
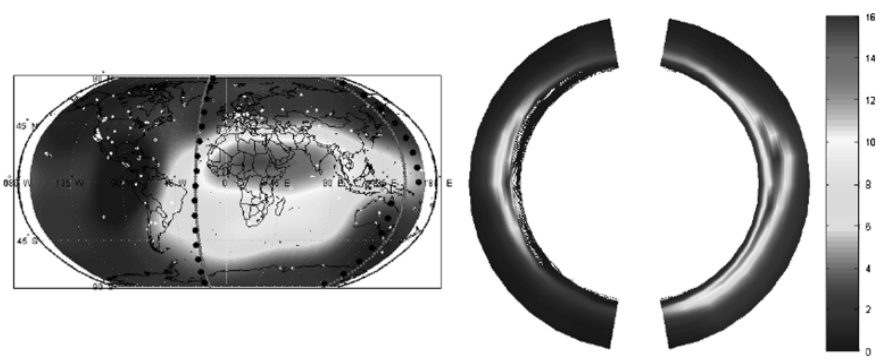

(b)
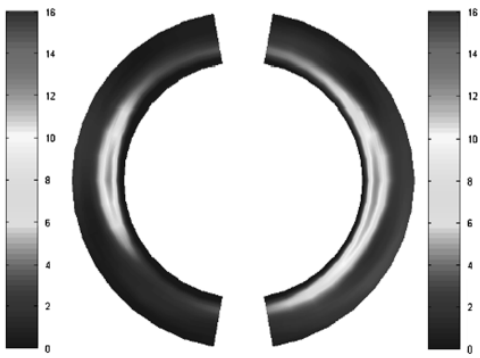

(d)
Fig. 3. (a) Vertical TEC from ED solution (orbit shown as red line) integrated from ground to $1000 \mathrm{~km}$ under the track of LEO satellite (black dots). Units are ED/1011 electrons $/ \mathrm{m}^{3}$. (b) Reference ring-slice ED from PIM. (Bottom) Solutions with an H-representation of order $n=8$ (204 unknowns, $a_{o}=10$ $\mathrm{km})$, with GNSS-R data. No ground data has been used. (c) No constrains $(0.15$ Tera el $/ \mathrm{m}^{3}$ mean error). (d) Solution with constraint (of $\lambda n^{2}$ type; $\lambda=500$ ) $\left(0.14 \mathrm{Tera} \mathrm{el} / \mathrm{m}^{3}\right.$ mean error).

TABLE I

VALUES OF GLOBLAL TEC IN ALL THE IONOSPHERE (FOURTH COLUMN), AND ED IN THE ORbital Ring (FIfTH COLUMN) AS SHOWn IN Fig. 3, USING DIFFERENT NUMBER OF COEFFICIENTS AND DIFFERENT VALUES FOR $a_{0}$. IN EACH COLUMN, We SHOW THE $n$-ORDER AND NUMBER OF COEFFICIENTS, THE $a_{0}$ PARAMETER, THE DATA Fit Residuals (DATA MinUs Model Residuals), AND the Ring Solution Fit (TRUth Minus Recovered SOlution), With/Without THE USE OF GNSS-R Data. All the Data EXCEPT ${ }^{(*)}$ Were COMPUTED USING 3 min OF DATA CADENCE. IN $(*)$ A 1-min Data CADENCE Was Used (IF Not, AND Without THE AdDITION OF CONSTRAINTS AND/OR GNSS-R DATA, SOLUTIONS WERE DEGENERATED, AS IN FIFTH AND Sixth Rows). FuRTHERMORE, No GROUND DATA WERE USED in This Particular Case. The Last Four Rows (Bold) Have Been Calculated Using a SMoothing Constraint

\begin{tabular}{l|l|l|l|l|l}
\hline $\mathrm{n}$ & $\#$ coeff & $a_{0}(\mathrm{~km})$ & $\begin{array}{l}\text { Fit Residuals } \\
(\text { TECU })\end{array}$ & \multicolumn{2}{l}{$\begin{array}{l}\text { Ring ED Error } \\
\left(\text { Tera el } / \mathrm{m}^{3}\right)\end{array}$} \\
\cline { 5 - 6 } & & & & With GNSS-R & $\begin{array}{l}\text { Without } \\
\text { GNSS-R }\end{array}$ \\
\hline 5 & 55 & 30 & 15.75 & 0.19 & 0.19 \\
\hline 6 & 91 & 10 & 11.35 & 0.35 & 0.45 \\
\hline 6 & 91 & 20 & 10.20 & 0.17 & 0.20 \\
\hline $8^{(*)}$ & 204 & 10 & 5.33 & 0.15 & 0.16 \\
\hline 8 & 204 & 10 & 7.26 & 0.28 & - \\
\hline 8 & 204 & 20 & 6.34 & 0.27 & - \\
\hline $\mathbf{8}$ & $\mathbf{2 0 4}$ & $\mathbf{1 0}$ & $\mathbf{1 2 . 2 2}$ & $\mathbf{0 . 1 8}(\lambda=\mathbf{5 0 0 0})$ & - \\
\hline $\mathbf{8}$ & $\mathbf{2 0 4}$ & $\mathbf{1 0}$ & $\mathbf{1 0 . 0 6}$ & $\mathbf{0 . 1 7}(\lambda=\mathbf{3 0 0 0})$ & - \\
\hline $\mathbf{8}$ & $\mathbf{2 0 4}$ & $\mathbf{1 0}$ & $\mathbf{8 . 0 6}$ & $\mathbf{0 . 1 5}(\boldsymbol{\lambda}=\mathbf{1 5 0 0})$ & - \\
\hline $\mathbf{8}$ & $\mathbf{2 0 4}$ & $\mathbf{1 0}$ & $\mathbf{7 . 2 9}$ & $\mathbf{0 . 1 5}(\lambda=\mathbf{5 0 0})$ & - \\
\hline
\end{tabular}

Finally, we recall that the spherical harmonic representation resolution is of the order of $180 / n$. Thus, for $n=8$, we are at about $22^{\circ}$ of spatial horizontal resolution and about $100 \mathrm{~km}$ vertical resolution.

At $n=14$, we would have about 1000 unknowns, a horizontal resolution of $13^{\circ}$, and about $50 \mathrm{~km}$ of vertical resolution. At $n=20$, we have almost 3000 unknowns, for $9^{\circ}$ of spatial resolution. This is in fact close to the resolution we actually 
used for the ED field generation with PIM. To these unknowns, the emitter-receiver biases (one per GPS satellite and GPS receivers) also need to be added (although we did not include them in our simulation for simplicity).

\section{CONCLUSION}

In this study, GNSS-R/O/N LEO and ground data were simulated based on the current GNSS-R code-ranging error budget, and a new 3-D tomographic representation used to study the impact of GNSS-R data for ionospheric ED tomography near a single LEO orbital ring.

The representation, which we call the H-representation, uses solutions similar to those of the hydrogen atom Schrödinger equation and provides an efficient way to represent the solution space. With as little as 55 coefficients $(n=5)$, a fit of 7 TECU was produced under the LEO track, using only LEO data, while the addition of IGS ground data gave a fit of about 13 TECU. Using more coefficients, and adding smoothing constraints, the solutions become more accurate, as expected.

The main point of this study is the demonstration that GNSS-R data improves the ED results in the LEO orbital ring area. It can be concluded from this study that the addition of GNSS-R data can cover an important gap over the oceans supporting occultation measurements, where ground data are not available. Future work is needed for more realistic simulations, to further improve GNSS-R error budgets and to understand ionospheric phase behavior-ideally through a GNSS-R mission to collect data from space in addition to further simulation, analysis, and air/ground experiments.

\section{ACKNOWLEDGMENT}

All Starlab authors have contributed significantly. The Starlab author list has been ordered randomly.

\section{REFERENCES}

[1] G. B. Arfken and H. J. Weber, Mathematical Methods for Physicists. New York: Academic, 1995.

[2] A. Komjathy and R. Langley, "Improvement of a global ionospheric model to provide ionospheric range error corrections for single-frequency GPS users," presented at the Proc. ION 52nd Annu. Meeting, Cambridge, MA, Jun. 19-21, 1996.

[3] P. Y. Le Traon, G. Dibarboure, G. Ruffini, and E. Cardellach. (2002, Dec.) Mesoscale ocean altimetry requirements and impact of GPS-R measurements for ocean mesoscale circulation mapping, Abridged Starlab report from the Paris Beta Project. [Online]. Available: http://arxiv.org/abs/physics/0212068. Courtesy of ESA/ESTEC and Starlab, 2003 update at http://starlab.es/gnssr2003/proceedings/ 18\%20P2\%20LeTraon.pdf. ESA/ESTEC Contract 15083/01/NL/MM
[4] S. Lowe, J. L. LaBrecque, C. Zuffada, L. J. Romans, L. Young, and G. A. Hajj, "First spaceborne observation of an earth-reflected GPS signal," Radioi Sci., vol. 37, no. 1, pp. 1-28, 2002.

[5] G. Ruffini et al.. Using GNSS reflections for ionospheric studies. presented at Space Weather Workshop, ESA-ESTEC. [Online]. Available: http://www.estec.esa.nl/wmwww/wma/spweather/workshops/ SPW_W3/PROCEEDINGS_W3/ruffini.PDF. See also Ruffini, G., and Soulat, F., "Paris interferometric processor theoretical feasibility study part I and Part II." http://arxiv.org/ps/physics/0011027.

[6] G. Ruffini and F. Soulat, "Paris interferometric processor theoretical feasibility study," presented at the 2nd European GNSS Surface Reflection Working Group (EGSR-2), Noordwijk, The Netherlands, Aug. 2000, PIPAER-IEEC-TN-1100/2100.

[7] G. Ruffini, L. Cucurull, A. Flores, and A. Rius, "A PIM-aided kalman filter for GPS tomography of the ionospheric electron content," Phys. Chem. Earth (C), vol. 24, no. 4, pp. 365-369, 1999.

[8] G. Ruffini, A. Flores, and A. Rius, "GPS tomography of the ionospheric electron content with a correlation functional," IEEE Trans. Geosci. Remote Sensing, vol. 36, no. 1, pp. 143-153, Jan. 1998.

[9] R. Shankar, The Principles of Quantum Mechanics. Norwell, MA: Kluwer, Aug. 1994.

[10] A. Tarantola, Inverse Problem Theory. Methods for Model Parameter Estimation. Philadelphia, PA: SIAM, 2004.

Josep Marco Pallarés was born in Barcelona, Spain, in 1978. He received the degree in physics from the University of Barcelona, Barcelona, in 2000, and the M.S. degree in neuroscience in 2002 . He is currently pursuing the Ph.D. degree in neuroscience at the University of Barcelona.

$\mathrm{He}$ is currently with Starlab, Barcelona, focusing on new mathematical tools in order to increase the spatial resolution of tomography (EEG and GNSS-R).

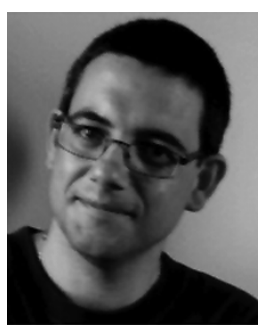

Giulio Ruffini was born in Barcelona, Spain, in 1966. He received the B.A. degrees in mathematics and physics from the University of California, Berkeley, in 1988, and the M.S. and Ph.D. degrees in physics from the University of California, Davis, in 1990 and 1995, respectively.

During the summers of 1990 to 1995 , he was a Graduate Researcher in theoretical physics at the Los Alamos National Laboratory, New Mexico. He then moved to IEEC in Barcelona, until September 2000. $\mathrm{He}$ is since working at Starlab Barcelona on GNSS-R.

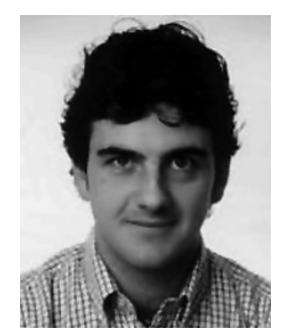

Leonardo Ruffini was born in Barcelona, Spain, in 1977. He graduated as an industrial engineer in 2001 from the Universitat Politècnica de Catalunya, Barcelona, specializing in mechanical design.

$\mathrm{He}$ is currently working as a Research Assistant (on GNSS-R data analysis) at Starlab, Barcelona. 